\title{
Neuroplasticity in Spinal Trauma: A Current Narrative Review of Treatments
}

\author{
Vinicius Benatti Freire1, Lucas Cressoni de Souza1, Mario Henrique de Lima Martinelli1, \\ Leonardo Moysés Sacchi'1, Thales Andolfo de Souza1, Antonio Eduardo Damin²
}

\author{
${ }^{1}$ Medical Students of the Nove de Julho University (Campus Vergueiro), São Paulo, Brazil \\ ${ }^{2}$ Department of Neurology of the Nove de Julho University Medical School, \\ Neurologist and Professor of Neurology at Nove de Julho University Medical School, São Paulo, Brazil \\ Email: viniciusbenattifreire@gmail.com, lucascressoni@gmail.com,damin@uni9.edu.br
}

How to cite this paper: Freire, V.B., Cressoni de Souza, L., Henrique de Lima Martinelli, M., Sacchi, L.M., Andolfo de Souza, T. and Damin, A.E. (2021) Neuroplasticity in Spinal Trauma: A Current Narrative Review of Treatments. World Journal of Neuroscience, 11, 91-107.

https://doi.org/10.4236/wjns.2021.112008

Received: February 25, 2021

Accepted: April 18, 2021

Published: April 21, 2021

Copyright $\odot 2021$ by author(s) and Scientific Research Publishing Inc. This work is licensed under the Creative Commons Attribution International License (CC BY 4.0).

http://creativecommons.org/licenses/by/4.0/ Open Access

\begin{abstract}
Neuroplasticity is a condition that is present from birth, being found in the central and peripheral nervous system, in both physiological and pathological terms. Based on the findings, therapeutic and non-therapeutic attempts were tested on spinal cord trauma to recover locomotor function below the level of the injury. The work defined and showed other forms of the term neuroplasticity, talk about some pathological and non-pathological conditions, and, finally, show neuroplasticity and some of its treatments in the spinal cord injury process. A narrative literature review from 2000 to 2020 of the PubMed platform was conducted and analysis of two books for the elaboration of this work. Animal/human studies were included that addressed pathologies, forms of treatment for spinal trauma, and qualis from B1 to A1. Pre-2000 articles, which addressed neuroplasticity only to understand the molecular mechanisms and articles that were not in English, were excluded. As a result, the main molecules and structures that inhibit neuroplasticity were found, and, based on their knowledge, forms of treatments were developed to inhibit these molecules and structures to assist in neuroplasticity and assist in possible functional recovery. It can be concluded that the physiological barriers are already being overcome by the most recent forms of treatment and that soon new studies may propose a form of treatment that is protocoled for all patients.
\end{abstract}

\section{Keywords}

Neuroplasticity, Spinal Cord Injury, Regeneration, Nanomaterials, Stem Cell, Pharmacology 


\section{Introduction}

\subsection{Definition, Forms, and Mechanisms of Neuroplasticity}

Neuroplasticity can be defined as the property of the nervous system to alter its function or structure in response to the environmental influences that affect it. One of the forms of neuronal plasticity is peripheral, also known as true regeneration, where the lost circuit and its function are reconstituted [1] [2]. This is perceived when in a surgical procedure there is a loss of sensation in the newly operated region, with sensitivity restored in a certain period. Another type of neuroplasticity is the functional, which was demonstrated in a study involving musicians and non-musicians, where the area of finger representation in these two groups was analyzed. This area was shown to be greater for musicians who performed their learning early during childhood. In contrast, musicians who have focal dystonia (a motor disorder caused by excessive motor practice with the fingers), present a fusion of the cortical representation of the fingers, which is probably the cause of the disorder, which is the result of poorly adaptive plasticity. Of maladaptive neuroplasticity, it is known as phantom limb syndrome where it would be possible to reconstruct an imaginary representation of the limb, in the brain [1]-[9].

\subsection{Plasticity and Axonic Regeneration}

Axonic plasticity is different from axonic regeneration since axons are preserved and can be modified without the need for injury, whereas in regeneration, axons are injured and there is a response process to this stimulus. This type of plasticity is the way the nervous system uses to consolidate neural circuits according to environmental influences. An example of axonic plasticity is amblyopia, which is the lack of visual acuity involving particularly stereoscopic vision [1] [2] [10].

\subsection{Spinal Cord Injury}

Neuroplasticity and its mechanisms are not only present in physiological situations but also in pathological ones. One of these forms is neuroplasticity that occurs in the CNS in a spinal cord injury. Spinal cord injuries occur between 250,000 and 500,000 people per year are caused by traumatic events. The complications known caused by spinal cord injury are due to two main pathophysiological events: the initial physical injury and the subsequent secondary injury initiated by the initial trauma. The primary injury is acute and caused by mechanical forces, such as compression and displacement, which can physically disrupt cell membranes and blood vessels and destroy many local neurons and glia. The primary mechanical injury induces a cascade of chemical reactions and biological events, leading to a secondary injury that occurs in the hours after the initial injury. These secondary injury processes cause oxidative damage by activated radical oxygen, damage mediated by calcium, by the influx of calcium ions, immune reactions, apoptosis, hemorrhage, inflammation, and edema, re- 
sulting in axonal degeneration, demyelination, and cavitation at the injury site. Also, spinal cord injury causes the death of elevated neurons and oligodendrocyte cells that result in positive regulation of axonal growth-inhibitory factors at the injury site [11] [12].

Spinal cord injury can be classified according to severity. The classification is graded as follows: ASIA A (level A), ASIA B (level B), ASIA C (level C), and ASIA D (level D). An ASIA A injury is when the patient has a complete loss of function below the level of the spinal cord injury, whereas in ASIA B, ASIA C, and ASIA D injuries, partial sensory preservation, partial motor preservation occurs and both functions are partially preserved. Most of the patients who are victims of spinal cord injury have residual edges of white substance spared on the peripheral margins of the spinal cord. This spared tissue usually constitutes a small proportion of the pre-injury white matter volume, between $1 \%$ to $10 \%$ of the original amount of tissue. The axons that were spared in this limit are hypomyelinated or demyelinated, indicating that both the remyelination of these axons and the plastic reorganization of their terminals below the injury site would be necessary to achieve functional recovery. When greater proportions of white matter are spared after the initial injury, patients may manifest several clinical syndromes related to ASIA B, ASIA C, or ASIA D [11].

Spontaneous improvement in function occurs in the vast majority of patients (60\% - 80\%), with partial preservation of motricity, supporting the theory that the spared projections can undergo spontaneous plasticity and can generate functional improvement in patients. Only $20 \%$ of ASIA A patients exhibit any spontaneous improvement in one year after the injury, indicating the potential ability of small edges of spared tissue to mediate functional improvement. However, patients with spinal cord injury classified as ASIA B, C, or D show very high rates of spontaneous improvement in function from the moment of the initial injury [11].

However, some patients have chronic lesion stages. Patients who have this type of stage, have little spontaneous improvement in their lost functions and drugs that intend to increase spontaneous plasticity may lose an opportunistic window that exists for limited periods after an acute injury [11].

\subsection{Treatments}

Among the pharmacological treatments, we have hydralazine, which has an antihypertensive effect and invades other tissues when administered by IV or oral route, abruptly reducing blood pressure, requiring a direct application to the lesion site so that it inhibits acrolein, which it is responsible for secondary nerve tissue damage, proving to be effective in treating spinal cord injury. The administration of clonidine was also shown to be efficient in the treatment of the most severe spinal injury, and its effects persisted after elimination of the drug. The interpretation of results and concluding were impaired after the application of doses of 4-aminopyridine, and its effects on the treatment of spinal cord injury 
are not clear. In contrast, treatment with GM-1 ganglioside was shown to be beneficial in patients with chronic spinal cord injury, and quite efficient in increasing motor scores after the application of GM-1 ganglioside. Studies with methylprednisolone, a synthetic steroid, showed that this drug reduces inflammation around the injury site by suppressing the immune system [12]-[19].

Other approaches consist of non-pharmacological actions that include stem cells, nanotechnology, hydrogel nanoparticles, and delivery of drugs by polymeric nanoparticles, scaffolding, and IN-1 antibody. For these forms of treatment, it was necessary to understand the molecular mechanisms of these approaches in some studies [12] [20] [21] [22] [23] [24].

Many studies have proven that the treatment involving hydrogel nanoparticles is effective for the process of regeneration of neurons, through progenitor cells, and of the axons at the injury site, due to the mechanical properties and structural architecture that make them a good option to present factors endogenous to the injured spinal cord. Also, polymeric nanoparticles, such as polylactic-co-glycolic acid (PLGA), have the characteristic of regulating the release of drugs from administration systems and at the same time provide an appropriate structure for the tissue and can protect anti-degradation drugs, in addition to increasing their stability. In addition, they were combined with growth factors or hybrid structures that incorporate tissue cells, favoring repair at the spinal cord injury site [12] [24]-[38].

The application of the IN-1 antibody directly to the lesion site was performed to inhibit a part of the central nervous system myelin, which when its substrate was activated inhibited the growth of neurites, such as the Nogo protein [39] [40] [41].

A component of the nanoconjugate, called WGA, was identified in the axons descending from the ipsilateral phrenic nerve motor neurons and in the rostral ventral respiratory group bilaterally through immunostaining techniques in the rats that used this treatment, but it was not possible to identify the theophylline in these locations, but it can be inferred that theophylline is present due to the recovery of phrenic nerve activity on the $2^{\text {nd }}$ day after application. Malondialdehyde and myeloperoxidase levels were lower with treatment and the superoxide dismutase enzyme, used by cells to promote antioxidation, was higher. The treatment was also able to attenuate local hemorrhages, increase the number of neurons, glial cells, and the formation of the glial junction at the injured site, and a very significant reduction in inflammatory cells, autophagosomes, and glial scar, showing a reduction in the post-injury repair period, confirmed histopathology. In another way of thinking, an experimental study involving gold nanoparticles wrapped in an extract of Juglans regia and the antiepileptic Zonisamide was carried out to measure the effectiveness of this form of treatment [42] [43].

It can be inferred based on these findings, that this form of treatment is very promising for patients with spinal cord injury. One of these studies associated 
agarose gel nanoparticles with the hormone 17ß-estradiol, forming the compound PLGA-E2 for the treatment of spinal cord injury and reduction of the toxic effects of the hormone in rats. The treatment increased concentrations of vascular endothelial growth factor, anti-inflammatory cytokines, and protein levels of axonal neurofilament. Treatment also reduced gliosis/microgliosis formation, astrocytic hypotrophy, decreased chondroitin sulfate proteoglycan, and microglial hyporeactivity in the lesion, in addition to partial functional recovery demonstrated by increased function in the hind limbs, increased frequency of strides, and partial recovery balance, the latter being assessed by gait symmetry [44] [45].

Another study involving the PLGA was also carried out for the treatment of spinal cord injury to propose a new method of delivery of antioxidant nanoparticles involving dogs. The limitations consisted in not carrying out additional tests for a more targeted treatment and with more control over the dosage of antioxidants and the complement system. This delivery strategy can also be considered for other molecules capable of promoting neuroprotective astrocytes, providing a specific therapeutic treatment for cells to improve SCI and other neurological diseases [46].

\section{Objective}

The objective of this work was to define the term neuroplasticity and show other forms of it, highlight some pathological and non-pathological conditions and, finally, demonstrate neuroplasticity and some of its treatments in the spinal cord injury process.

\section{Materials and Methods}

To carry out this narrative review work, the PubMed platform was used to search review articles and the originals on the subjects of neuroplasticity, central and peripheral, neurogenesis, spinal cord injury, as well as the treatments that were carried out for the pathology. Only Articles from 2000 to 2020 were analyzed, articles before this date were removed, as well as articles that were not in English. Furthermore articles which addressed neuroplasticity only to understand the molecular mechanisms, but not the clinical effects of the treatments, were removed. Studies in animals/humans, which addressed pathologies, the forms of treatment for spinal trauma, and with qualis from B1 to A1 were included.

In addition to these means of research, two books were also used as the basis for its composition, namely: Neuroscience of Mind and Behavior 1st Edition, Rio de Janeiro: Guanabara Koogan, 2008 (Roberto Lent-Coordinator) and Neuroscience Third Edition, United States of America: Sinauer Associates 2004, in chapters 23 and 24 (Edition by Dale Purves et al.). The descriptors used were: Neuroplasticity, Neurogenesis, Spinal Cord Injury, Regeneration, Secondary Injury, Nanomaterials, Stem Cell, Nanoparticles, Side Effects, Medicines. 


\section{Results and Discussion}

The studies evaluated by the review show that after spinal cord trauma, several molecules are released as a result of the trauma, triggering a secondary spinal cord injury, being characterized as a chemical injury arising from oxidative stress. An example of a molecule is acrolein, which is released after lipid peroxidation of the myelin sheath after trauma, which is cytotoxic, among others. Some structures are also formed at the site and end up preventing neuroplasticity that would culminate in regeneration for functional recovery below the level of the injury [41] [14] [12]. Table 1 shows some of the main molecules and structures that are neuroinhibitory.

A greater understanding of the inhibitory mechanisms that occur during the neuroplasticity process is also necessary to propose an adequate treatment method, given the complexity of these mechanisms. However, some main forms were studied to seek a functional recovery below the level of the injury. Therefore, we show below some forms of treatment for spinal cord injury.

Many drug tests have been carried out to promote adequate neuroplasticity to obtain true regeneration in the injured site for a functional recovery below the level of the injury. Studies involving clonidine, cyproheptadine, levodopa, 4-aminopyridine, GM-1 ganglioside, baclofen, and methylprednisolone have been carried out and reviewed for this purpose [13] [16] [17] [18] [19] [47]. Hydralazine has an antihypertensive effect and invades other tissues when administered via IV or orally, abruptly reducing blood pressure, requiring the direct application to the injury site to inhibit acrolein, which is responsible for nervous system secondary tissue damage, proving effective in the treatment. The administration of clonidine has also been shown to be efficient in the treatment

Table 1. Main neuroinhibitory molecules and structures.

\begin{tabular}{|c|c|}
\hline Structure/Molecule & Inhibitory Function \\
\hline Glial Scar & $\begin{array}{l}\text { Inhibitory structure that acts as an absolute barrier } \\
\text { for regenerating axons. }\end{array}$ \\
\hline $\begin{array}{c}\text { Chondroitin Sulfate Proteoglycans (CSPGs), } \\
\text { Ephrins, Semaphorins }\end{array}$ & $\begin{array}{c}\text { Glial scar tissue molecules that inhibit axonal } \\
\text { growth. }\end{array}$ \\
\hline Neurocan, Versican, NG2 & $\begin{array}{l}\text { Proteins present in CSPGs that are regulated } \\
\text { positively after injury to the Central Nervous } \\
\text { System in order to block axonal regeneration. }\end{array}$ \\
\hline Sulfated glycosaminoglycan chains & Inhibit axon growth, largely by GTPase Rho. \\
\hline Meningeal cells in CNS injuries & $\begin{array}{c}\text { Main component of the lesion nucleus that acts as } \\
\text { a barrier to axonal growth. }\end{array}$ \\
\hline Myelin & $\begin{array}{l}\text { Second largest source of axonal growth inhibitory } \\
\text { molecules and stimulated by oligodendrocytes. }\end{array}$ \\
\hline $\begin{array}{c}\text { NogoA, Myelin-Associated Glycoprotein } \\
\text { (MAG), CSPG versican, Oligodendrocyte } \\
\text { myelin glycoprotein (OMgp), Semaphorin 4D }\end{array}$ & $\begin{array}{l}\text { Molecules identified in myelin that lead to axonal } \\
\text { inhibition via receptors, such as the Nogo receptor. }\end{array}$ \\
\hline
\end{tabular}

Source: Made by analyzing the study by Fawcett, 2006. 
of the most severe spinal injury, and its effects persisted after elimination of the drug. Cyproheptadine has also benefited patients with more severe spinal cord injuries, facilitating locomotor initiation or training by reducing severe spasticity and muscle clonus, as well as clonidine. Levodopa treatment associated with locomotor training proved to be efficient in changes in voluntary muscle strength, walking function, and activities of daily living, and when compared to placebo, which was also associated with locomotor training, there was no difference in improvement. The interpretation of results and concluding were impaired after the application of doses of 4-aminopyridine, and its effects on the treatment of spinal cord injury are not clear. In contrast, treatment with GM-1 ganglioside was shown to be beneficial in patients with chronic spinal cord injury, and quite efficient in increasing motor scores after the application of GM-1 ganglioside. Studies with methylprednisolone, a synthetic steroid, showed that this drug reduces inflammation around the injury site by suppressing the immune system, in addition to having a neuroprotective effect. Evidence of the efficacy of baclofen treatment is limited, but an increase in the gait scores of the Functional Independence Measure (FIM) after the use of baclofen has been proven, but with small changes in walking [12]-[19]. Table 2 shows a summary of the mentioned forms of treatment.

Most pharmacological approaches are efficient in improving motor and sensory capacity below the level of the lesion, but some side effects and limitations concerning the use of monoaminergic drugs (Clonidine, Ciproheptadine, and Levodopa) and the others mentioned above have been evidenced. Showing that

Table 2. Pharmacological treatments mentioned.

\begin{tabular}{|c|c|c|}
\hline Authors/Year & Treatment & Result/Observations \\
\hline $\begin{array}{l}\text { van der Bruggen et al., } 2001 \\
\text { DeForge et al., } 2004 \\
\text { Domingo et al., } 2012\end{array}$ & 4-aminopyridine & There was not benefit. \\
\hline $\begin{array}{l}\text { Maric, Zorner and Dietz, } 2008 \\
\text { Domingo et al., } 2012\end{array}$ & Levodopa & $\begin{array}{l}\text { No difference between placebo and } \\
\text { medication. }\end{array}$ \\
\hline Domingo et al., 2012 & $\begin{array}{l}\text { Clonidine } \\
\text { Cyproheptadine }\end{array}$ & $\begin{array}{l}\text { Improvement in walking scores. } \\
\text { Walking speed increased; Improved } \\
\text { muscle coordination; Decreased clonus. }\end{array}$ \\
\hline & $\begin{array}{l}\text { Cyproheptadine }+ \\
\text { Clonidine }\end{array}$ & $\begin{array}{l}\text { Decreased clonus; Muscle activity of the } \\
\text { lower limbs increased. }\end{array}$ \\
\hline $\begin{array}{c}\text { Geisler et al., } 2001 \\
\text { Domingo et al., } 2012\end{array}$ & Ganglioside GM-1 & $\begin{array}{l}\text { Increased motor scores; Walking } \\
\text { distance and speed increased. }\end{array}$ \\
\hline Domingo et al., 2012 & Baclofen & Improves motor scores. \\
\hline $\begin{array}{c}\text { Qian et al., } 2005 \\
\text { Domingo et al., } 2012\end{array}$ & Methylprednisolone & $\begin{array}{l}\text { Neuroprotective; Anti-inflammatory; } \\
\text { Attenuation of lipid peroxidation; }\end{array}$ \\
\hline Nejati-Koshki K., et al., 2017 & & Myopathies and other complications. \\
\hline $\begin{array}{l}\text { Liu-Snyder, P. et al., } 2006 \\
\text { Hamann K. et al., } 2007 \\
\text { Hamann, K. \& Shi, R., } 2009\end{array}$ & Hydralazine & $\begin{array}{l}\text { Neuroprotection against acrolein; } \\
\text { Injury reversal. }\end{array}$ \\
\hline
\end{tabular}


at times it was more harmful than beneficial to patients [13] [15] [16] [17] [47].

Side effects of monoaminergic medications ranged from mild to severe, consisting of xerophthalmia, increased urinary frequency, fatigue, dizziness, constipation, decreased urination urgency, increased appetite, numbness, altered sexual function, nausea/vomiting, headache; and subjective decrease in headache, strength, skin rash, vertigo and dry mouth in several patients who were treated with these drugs. However, these side effects are not only reported when using monoaminergic drugs. The use of 4-aminopyridine culminated in the development of nausea, dizziness, and difficulty sleeping in $50 \%$ of the patients, and, also, there was withdrawal due to severe side effects (dizziness, weakness, and regression in walking ability), intolerance to $40 \mathrm{mg}$ doses of the drug and development of transient symptoms (dizziness, headache and flu-like symptoms). The use of Baclofen demonstrated symptoms of overdose in high doses resulting in the hospitalization of patients, however, this side effect was found in the minority of them. In contrast, no side effects were reported in the use of Ganglioside GM-1 [17] [18] [19] [47].

Studies involving hydralazine and methylprednisolone have also demonstrated side effects and limitations that are different from those already mentioned. The proper concentration of Hydralazine may not be achieved in vivo, since after administration of the drug intravenously, a peak concentration equivalent to $0.5-1.0 \mu \mathrm{M}$ is expected, with a half-life of $30-60$ minutes. In addition, acrolein concentrations are much higher than the peak hydralazine concentration, not being sufficient to neutralize acrolein. The drug can also induce vasodilation due to its antihypertensive mechanism, a non-beneficial effect in patients with spinal cord injury, as they are suffering neurogenic shock, in addition to the drastic decrease in blood pressure involving the guanylate cyclase receptor that is coupled to protein $\mathrm{G}$, in smooth muscle. Another problem involving Hydralazine is in the development of autoimmune disease (Lupus Erythematosus-modification in DNA, developing antinuclear antibody) and in the accumulation of acrolein-protein adducts captured by hydralazine in the body, which the effects of this accumulation are not clear [12] [15] [48].

The use of methylprednisolone, despite having positive effects with studies in the late twentieth century, proved to be harmful to patients with prolonged use and in high doses. Qian and colleagues conducted a study in which they used Methylprednisolone as a form of treatment. The study applied the protocol recommended by the NASCIS III trial, which recommends specific doses of methylprednisolone over certain periods. After its use, it was evidenced that the patients developed acute corticosteroid myopathy. Besides, other complications have been reported and consisted of gastric bleeding, sepsis, pneumonia, and wound infections [12] [13] [16].

Based on the side effects and limitations previously reported, other approaches for the treatment of spinal cord injury have been developed, consisting of non-pharmacological actions such as stem cell therapy, nanotechnology, nano- 
particles (mainly hydrogel), polymeric, and others that provide delivery medication in a controlled/targeted manner and medullary supports. In addition, a monoclonal antibody, called IN-1, was found to prevent the inhibitory effects of Nogo, being present only in the central nervous system. For these forms of treatment, it was necessary to understand the molecular mechanisms of these approaches in some studies [12] [20] [21] [22] [23] [24] [39].

The treatment with stem cells in the spinal cord regeneration process proved to be efficient, since these cells replaced the nerve cells killed as a result of the injury, also, they promote the inhibition of defense cells ( $\mathrm{T}$ lymphocytes, macrophages, and microglia) that contribute to secondary spinal cord injury, secrete trophic factors essential for neural growth (such as Brain-derived neurotrophic fator (BDNF); Glial cell line-derived neurotrophic fator (GDNF); Insulin-like growth fator (IGF); Vascular endothelial growth fator (VEGF); Fibroblast growth factor-2 (FGF-2); and Transforming growth fator (TGF)), increase serum IL-10 and decrease TNF tumor necrosis factor-a serum. Also, the association of stem cells with superparamagnetic iron oxide nanoparticles (SPIONPs) facilitates the homing of stem cells at the injury site. Treatment involving nanotechnology consists of the ability of nanomaterials to increase bioavailability and circulation time at the injury site, proven with methylprednisolone and hydralazine. Many studies have proven that the treatment involving hydrogel nanoparticles is effective for the process of regeneration of neurons, through progenitor cells, and of the axons at the injury site, due to the mechanical properties and structural architecture that make them a good option to present factors endogenous to the injured spinal cord. Also, polymeric nanoparticles, such as PLGA, have the characteristic of regulating the release of drugs from administration systems and at the same time provide an appropriate structure for the tissue and can protect drugs against degradation, as well as increase its stability. Other nanoparticles were studied, such as iron oxide, ferulic acid (FA), and glycolchitosan (GC), and it was observed that the former favors the homing of mesenchymal stem cells at the site of spinal cord injury, in a mouse model with spinal cord injury, and that the last two are natural neuroprotectors, favoring useful recovery after spinal cord injury. Treatment with spinal cord supports has been studied in rats and it has been found that these supports can bind to immobilized signaling molecules or drugs for controlled administration of therapeutic agents, improve axonal regeneration, provide an appropriate site for cell adhesion and migration transplanted cells, regulate the local microenvironment and protect neural progenitor cells (NPCs) from the inhibitory effects of CNS myelin after spinal cord injury. In addition, they were combined with growth factors or hybrid structures that incorporate tissue cells, favoring repair at the spinal cord injury site [12] [24]-[38].

The monoclonal antibody IN-1 was located only in the central nervous system to inhibit a part of the myelin that it has to inhibit Nogo to provide an adequate growth of neurites in the injured site in any part of the central nervous system. 
After performing applications of IN-1 at the spinal cord injury site, in the corticospinal tract, there was an improved regeneration observed, as well as extensive sprouting, in addition to anatomical plasticity. Such results were also found in spinal axons [39] [40] [41]. Table 3 shows a summary of these forms of treatment that were mentioned above.

No limitations were reported regarding the use of these products, however, concerning spinal supports, some limitations were taken into account. Depending on the material, the cord support may not be efficient. Nejati-Koshiki and colleagues analyzed the most common spinal supports used to treat spinal cord injuries, separating them into natural and synthetic materials. The main natural products consist of agarose, alginate, chitosan, fibrin, fibronectin, and methylcellulose, while the main synthetics are tubes (PGA/PLA/PLGA/PLCL), Matigrel and PEG/PEo [12].

Table 3. Non-pharmacological treatments mentioned.

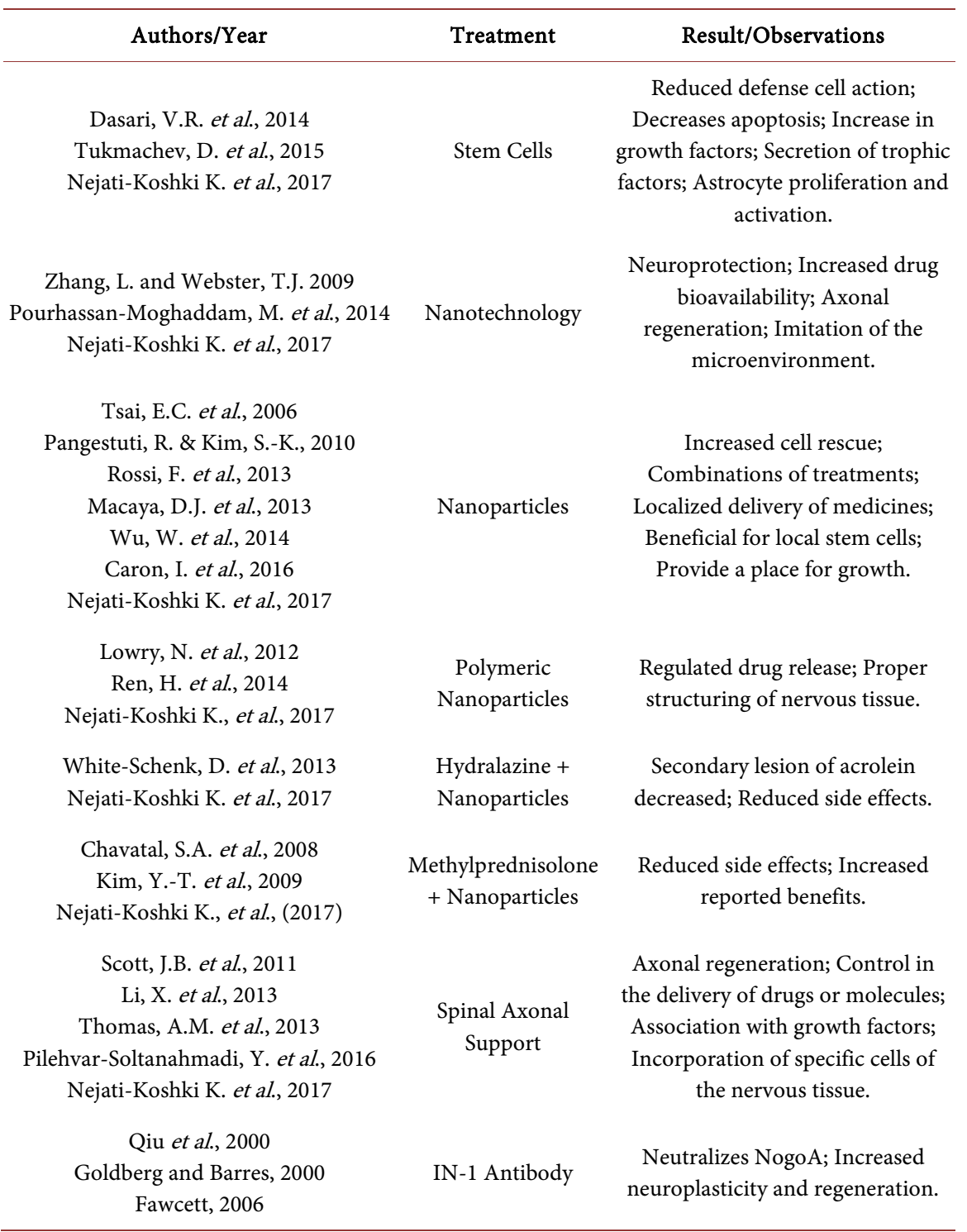


The main limitations of natural products are problems in the biodegradation of the material, which may be rapid or nonexistent (Agarose, Fibronectin, and Methylcellulose), the fragility of the support itself (Fibrin), and problems with its own regeneration/neuroplasticity (Alginate and Chitosan). The limitations of synthetic materials are because they are not biodegradable (PEG/PEo) and do not provide adequate axonal growth (PGA/PLA/PLGA/PLCL and Matrigel) [12].

Other synthetic polymers, in addition to those already reported, are being studied to be used to the fullest in the field of biomedicine. Some of them such as Poli ( $\alpha$-hydroxy esters), polyanhydride polymers, polycaprolactone, and poly (propylene fumarate) is being tested or manufactured because they are good candidates for the controlled delivery of medicines in medicine, in addition to their possible use for engineering purposes of fabrics, such as polyurethane. They have different degradations and compositions, and some have their main limitations related to their degradation, such as polycaprolactone which has a very slow degradation, and polyvinyl alcohol which presents incomplete degradation, but which has characteristics that make its transformation into hydrogels easier. However, some stand out positively in the field of degradation, such as polyurethane, which has several patterns of degradation, including hydrolysis, oxidation, and enzymatic processes. In addition to degradation, there are other obstacles found in some of these polymers, such as the difficult handling of poly (propylene fumarate) at room temperature and polyanhydride polymers that have limited life, high reactivity, and instabilities as main deficiencies. No other limitations were found in handling, degradation, and attributes. Also, no side effects of these polymers have been reported [49].

More recent studies involving nanotechnology have used more sophisticated treatments with other drugs. Rat models were used to demonstrate functional recovery of the phrenic nerve after hemisection of the cervical spinal cord using nanoconjugates (agglutinin + horseradish peroxidase + gold nanoparticles) associated with theophylline to aid in the process. A component of the nanoconjugate, called WGA, was identified in the axons descending from the ipsilateral phrenic nerve motor neurons and in the rostral ventral respiratory group bilaterally through immunostaining techniques in the rats that used this treatment, but it was not possible to identify the theophylline in these locations, but it can be inferred that theophylline is present due to the recovery of phrenic nerve activity on the $2^{\text {nd }}$ day after application [42].

Another study involving rodent models aimed to inhibit the Nogo protein receptor in the axons and promote a possible functional recovery using methylprednisolone (MP). To carry out the work, human albumin serum was used to form nanoparticles (NPs), which were conjugated to the peptide NEP1-40 (antagonist of the Nogo axonal receptor), thus forming the NEP1-40-MP-NP complex. The levels of malondialdehyde (indicates lipid peroxidation) and myeloperoxidase (an indicator of the accumulation of polymorphonuclear leukocytes) 
were lower with the treatment and the superoxide dismutase enzyme, used by the cells to promote antioxidation, were higher. The treatment was also able to attenuate local hemorrhages, increase the number of neurons, glial cells, and the formation of the glial junction at the injured site, and a very significant reduction in inflammatory cells, autophagosomes, and glial scar, showing a reduction in the post-injury repair period, which confirmed histopathology. Also, the use of this complex to restore bone microarchitecture and significantly increase locomotor scores was observed [43].

In another way of thinking, an experimental study involving gold nanoparticles (AuNPs) wrapped in an extract of Juglans regia and the antiepileptic Zonisamide was carried out to measure the effectiveness of this form of treatment. The study showed that the drug release is very controlled and very stable. This form of release mediated by gold nanoparticles proved to be more cytotoxic to CTX TNA 2 cells (astrocyte type 1, with fibroblast morphology) compared to the free drug in the bloodstream. In addition, the AuNPs wrapper does not use any form of chemicals that can cause systemic toxicity. Based on these findings, it can be inferred that this form of treatment is very promising for patients with spinal cord injury [50].

In 2020, three studies were published discussing new perspectives. One of these studies associated agarose gel nanoparticles (PLGA) with the hormone 17ß-estradiol (E2), forming the compound PLGA-E2 for the treatment of spinal cord injury and reduction of the toxic effects of the hormone in rats. The treatment increased concentrations of vascular endothelial growth factor (VEGF), anti-inflammatory cytokines (IL-4, IL-10, and IL-13), and axonal neurofilament protein (NFP) levels. In addition, there was an increase in glutamine synthetase, reducing the excess of glutamate and ammonia to promote neuroprotection, and the glial cell-derived neurotrophic factor (GNDF), also causing neuroprotection and sustaining axonal regeneration. The treatment also provided a reduction in the formation of gliosis/microgliosis, astrocytic hypotrophy, a decrease in chondroitin sulfate proteoglycan (CSPG), and microglial hyporeactivity in the lesion. In addition to partial functional recovery demonstrated by increased function in the hind limbs, an increase in the frequency of strides, and partial recovery of balance, the latter being assessed by gait symmetry [44].

Another study involving PLGA was also carried out for the treatment of spinal cord injury to propose a new method of delivery of antioxidant nanoparticles involving dogs. Instead of using medications, two enzymes were used to decrease oxidative stress-mediated by reactive oxygen species (ROS). In the nanoparticles, two enzymes have been internalized that are capable of reducing ROS which is Superoxide Dismutase (SOD), catalyzing superoxide $\left(\mathrm{O}_{2}^{-}\right)$in oxygen $\left(\mathrm{O}_{2}\right)$ and hydrogen peroxide $\left(\mathrm{H}_{2} \mathrm{O}_{2}\right)$, and Catalase, which degrades $\mathrm{H}_{2} \mathrm{O}_{2}$. Despite avoiding secondary spinal cord injury mediated by ROS and cytokines, many side effects were evident and reported in the study, in addition to limitations as well. Side effects ranged from mild to severe, affecting various body systems. Such as bra- 
dycardia, severe hypotension, weakness, hyper-salivation, involuntary urination, defecation, hyper or systemic hypotension, changes in blood count (leukopenia-with subsequent leukocytosis, and thrombocytopenia-anemia and increased RDW), skin flushing, and elevated alanine transferase (ALT). Many of these side effects were compatible with complement activation-related pseudo-allergy syndrome (CARPA). The limitations consisted in not carrying out additional tests for a more targeted treatment and with more control over the dosage of antioxidants and the complement system [45].

A promising study published in January 2020 demonstrated the selective efficacy of an anti-inflammatory called Rolipram delivered by biodegradable hydrogel (NG) nanoparticles in limiting the pro-inflammatory response mediated by astrocyte activation in a rat model with spinal cord injury (SCI), but other diseases with a glial response based on astrocytes can also gain from this selective therapeutic approach. This delivery strategy can also be considered for other molecules capable of promoting neuroprotective astrocytes (phenotype A2), providing a specific therapeutic treatment for cells to improve SCI and other neurological diseases. When tested intensively in a rat model with spinal cord injury, there was an improvement in motor performance, but only in the initial phase after the injury, reducing astrocytosis and preserving neuronal cells [46]. Table 4 shows a summary of recent forms of treatment.

\section{Conclusion}

The physiological barriers that prevent neuroplasticity are already being overcome by advances in the treatment of spinal cord injury. Combined treatment (pharmacological and non-pharmacological) is the most appropriate way for the functional recovery of patients with spinal cord injury because they act differently

Table 4. Recent treatments mentioned.

\begin{tabular}{|c|c|c|}
\hline Authors/Year & New treatments & Result/Observations \\
\hline Liu et al., 2019 & $\begin{array}{l}\text { Agglutinin }+ \text { Horseradish peroxidase } \\
+ \text { Gold nanoparticles }+ \text { Theophylline }\end{array}$ & $\begin{array}{l}\text { Functional recovery of the phrenic } \\
\text { nerve. }\end{array}$ \\
\hline Lin et al., 2019 & $\begin{array}{l}\text { Albumin nanoparticle + NEP1-40 } \\
\text { peptide + Methylpredinisolone }\end{array}$ & $\begin{array}{l}\text { Neuroprotection, anti-inflammation, } \\
\text { neuroproliferation, antioxidation and } \\
\text { reversal of damage from the primary } \\
\text { lesion. }\end{array}$ \\
\hline Fang et al., 2019 & AuNP + Juglans regia + Zonisamide & $\begin{array}{l}\text { Selective cytotoxicity, high stability } \\
\text { and controlled release. }\end{array}$ \\
\hline Cox et al., 2020 & Nanoparticles of PLGA + E2 & $\begin{array}{l}\text { Reduction of gliosis and } \\
\text { neuroinhibitory factors and increase } \\
\text { of growth factors and } \\
\text { anti-inflammatory cytokines. }\end{array}$ \\
\hline Robinson et al., 2020 & $\begin{array}{c}\text { PLGA nanoparticles + SOD + } \\
\text { Catalase }\end{array}$ & $\begin{array}{l}\text { Reduction of chemical damage and } \\
\text { significant systemic side effects. }\end{array}$ \\
\hline Vismara et al., 2020 & NG + Rolipram & $\begin{array}{l}\text { Selective cell action (A2) and } \\
\text { increased motor performance. }\end{array}$ \\
\hline
\end{tabular}


in different injury sites. In the near future, more studies are expected to be published and combined treatment may be filed for patients with spinal cord injury. If this occurs, access to treatments should be free and patented for less cost in order to improve the quality of life of all patients with spinal cord injuries.

\section{Conflicts of Interest}

The authors declare no conflicts of interest regarding the publication of this paper.

\section{References}

[1] Lent, R. (2008) Neuroscience of the Mind and Behavior. Guanabara Koogan S.A., Rio de Janeiro.

[2] Purves, D., et al. (2004) Neuroscience. 3rd Edition, Sinauer Associates Inc., Massachusetts.

[3] Ramachandran, V.S. and Rogers-Ramachandran, D. (2000) Phantom Limbs and Neural Plasticity. Archives of Neurology, 57, 317-320.

https://doi.org/10.1001/archneur.57.3.317

[4] Münte, T.F., Altenmüller, E. and Jäncke, L. (2002) The Musician's Brain as a Model of Neuroplasticity. Nature Reviews. Neuroscience, 3, 473-478. https://doi.org/10.1038/nrn843

[5] Fields, R.D. (2005) Myelination: An Overlooked Mechanism of Synaptic Plasticity? The Neuroscientist. A Review Journal Bringing Neurobiology, Neurology and Psychiatry, 11, 528-531. https://doi.org/10.1177/1073858405282304

[6] Sadato, N. (2005) How the Blind "See" Braille: Lessons from Functional Magnetic Resonance Imaging. The Neuroscientist. A Review Journal Bringing Neurobiology, Neurology and Psychiatry, 11, 577-582. https://doi.org/10.1177/1073858405277314

[7] Lledo, P.M., Alonso, M. and Grubb, M.S. (2006) Adult Neurogenesis and Functional Plasticity in Neuronal Circuits. Nature Reviews. Neuroscience, 7, 179-193. https://doi.org/10.1038/nrn1867

[8] Hensch, T.K. (2005) Critical Period Plasticity in Local Cortical Circuits. Nature Reviews. Neuroscience, 6, 877-888. https://doi.org/10.1038/nrn1787

[9] Gage, F.H. (2000) Mammalian Neural Stem Cells. Science (New York, N.Y.), 287, 1433-1438. https://doi.org/10.1126/science.287.5457.1433

[10] Hickmott, P.W. and Ethell, I.M. (2006) Dendritic Plasticity in the Adult Neocortex. The Neuroscientist. A Review Journal Bringing Neurobiology, Neurology and Psychiatry, 12, 16-28. https://doi.org/10.1177/1073858405282417

[11] Blesch, A. and Tuszynski, M.H. (2009) Spinal Cord Injury: Plasticity, Regeneration and the Challenge of Translational Drug Development. Trends in Neurosciences, 32, 41-47. https://doi.org/10.1016/j.tins.2008.09.008

[12] Nejati-Koshki, K., Mortazavi, Y., Pilehvar-Soltanahmadi, Y., Sheoran, S. and Zarghami, N. (2017) An Update on Application of Nanotechnology and Stem Cells in Spinal Cord Injury Regeneration. Biomedicine \& pharmacotherapy, 90, 85-92. https://doi.org/10.1016/j.biopha.2017.03.035

[13] Qian, T., Guo, X., Levi, A.D., Vanni, S., Shebert, R.T. and Sipski, M.L. (2005) High-Dose Methylprednisolone May Cause Myopathy in Acute Spinal Cord Injury Patients. Spinal Cord, 43, 199-203. https://doi.org/10.1038/sj.sc.3101681

[14] Liu-Snyder, P., Borgens, R.B. and Shi, R. (2006) Hydralazine Rescues PC12 Cells 
from Acrolein-Mediated Death. Journal of Neuroscience Research, 84, 219-227. https://doi.org/10.1002/jnr.20862

[15] Hamann, K. and Shi, R. (2009) Acrolein Scavenging: A Potential Novel Mechanism of Attenuating Oxidative Stress Following Spinal Cord Injury. Journal of Neurochemistry, 111, 1348-1356. https://doi.org/10.1111/j.1471-4159.2009.06395.x

[16] Domingo, A., et al. (2012) A Systematic Review of the Effects of Pharmacological Agents on Walking Function in People with Spinal Cord Injury. Journal of Neurotrauma, 29, 865-879. https://doi.org/10.1089/neu.2011.2052

[17] DeForge, D., Nymark, J., Lemaire, E., Gardner, S., Hunt, M., Martel, L., Curran, D. and Barbeau, H. (2004) Effect of 4-Aminopyridine on Gait in Ambulatory Spinal Cord Injuries: A Double-Blind, Placebo-Controlled, Crossover Trial. Spinal Cord, 42, 674-685. https://doi.org/10.1038/sj.sc.3101653

[18] Geisler, F.H., Coleman, W.P., Grieco, G., Poonian, D. and Sygen Study Group (2001) The Sygen Multicenter Acute Spinal Cord Injury Study. Spine, 26, S87-S98. https://doi.org/10.1097/00007632-200112151-00015

[19] Maric, O., Zörner, B. and Dietz, V. (2008) Levodopa Therapy in Incomplete Spinal Cord Injury. Journal of Neurotrauma, 25, 1303-1307. https://doi.org/10.1089/neu.2008.0583

[20] Zhang, L. and Webster, T.J. (2009) Nanotechnology and Nanomaterials: Promises for Improved Tissue Regeneration. Nano Today, 4, 66-80.

https://doi.org/10.1016/j.nantod.2008.10.014

[21] Pourhassan-Moghaddam, M., et al. (2014) Watercress-Based Gold Nanoparticles: Biosynthesis, Mechanism of Formation and Study of Their Biocompatibility in $\mathrm{Vi}$ tro. Micro \& Nano Letters, 9, 345-350. https://doi.org/10.1049/mnl.2014.0063

[22] Kim, Y.T., Caldwell, J.M. and Bellamkonda, R.V. (2009) Nanoparticle-Mediated Local Delivery of Methylprednisolone after Spinal Cord Injury. Biomaterials, 30, 2582-2590. https://doi.org/10.1016/j.biomaterials.2008.12.077

[23] Chavatal, S.A., Kim, Y.T., Bratt-Leal, A.M., Lee, H. and Bellamkonda, R.V. (2008) Spatial Distribution and Acute Anti-Inflammatory Effects of Methylprednisolone after Sustained Local Delivery to the Contused Spinal Cord. Biomaterials, 29, 1967-1975. https://doi.org/10.1016/j.biomaterials.2008.01.002

[24] White-Schenk, D., Shi, R. and Leary, J.F. (2013) Mesoporous Silica Nanoparticles for Treating Spinal Cord Injury. Proceedings SPIE 8587, Imaging, Manipulation, and Analysis of Biomolecules, Cells, and Tissues XI, San Francisco, 22 February 2013, 1-12. https://doi.org/10.1117/12.2004916

[25] Tsai, E.C., Dalton, P.D., Shoichet, M.S. and Tator, C.H. (2006) Matrix Inclusion within Synthetic Hydrogel Guidance Channels Improves Specific Supraspinal and Local Axonal Regeneration after Complete Spinal Cord Transection. Biomaterials, 27, 519-533. https://doi.org/10.1016/j.biomaterials.2005.07.025

[26] Rossi, F., et al. (2013) Tunable Hydrogel-Nanoparticles Release System for Sustained Combination Therapies in the Spinal Cord. Colloids and Surfaces B, Biointerfaces, 108, 169-177. https://doi.org/10.1016/j.colsurfb.2013.02.046

[27] Caron, I., et al. (2016) A New Three Dimensional Biomimetic Hydrogel to Deliver Factors Secreted by Human Mesenchymal Stem Cells in Spinal Cord Injury. Biomaterials, 75, 135-147. https://doi.org/10.1016/j.biomaterials.2015.10.024

[28] Macaya, D.J., Hayakawa, K., Arai, K. and Spector, M. (2013) Astrocyte Infiltration into Injectable Collagen-Based Hydrogels Containing FGF-2 to Treat Spinal Cord Injury. Biomaterials, 34, 3591-3602. 
https://doi.org/10.1016/j.biomaterials.2012.12.050

[29] Scott, J.B., Afshari, M., Kotek, R. and Saul, J.M. (2011) The Promotion of Axon Extension in Vitro Using Polymer-Templated Fibrin Scaffolds. Biomaterials, 32, 4830-4839. https://doi.org/10.1016/j.biomaterials.2011.03.037

[30] Pilehvar-Soltanahmadi, Y., Akbarzadeh, A., Moazzez-Lalaklo, N. and Zarghami, N. (2016) An Update on Clinical Applications of Electrospun Nanofibers for Skin Bioengineering. Artificial Cells, Nanomedicine, and Biotechnology, 44, 1350-1364. https://doi.org/10.3109/21691401.2015.1036999

[31] Li, X., et al. (2013) Promotion of Neuronal Differentiation of Neural Progenitor Cells by Using EGFR Antibody Functionalized Collagen Scaffolds for Spinal Cord Injury Repair. Biomaterials, 34, 5107-5116. https://doi.org/10.1016/j.biomaterials.2013.03.062

[32] Thomas, A.M., et al. (2013) Channel Density and Porosity of Degradable Bridging Scaffolds on Axon Growth after Spinal Injury. Biomaterials, 34, 2213-2220. https://doi.org/10.1016/j.biomaterials.2012.12.002

[33] Lowry, N., et al. (2012) The Effect of Long-Term Release of Shh from Implanted Biodegradable Microspheres on Recovery from Spinal Cord Injury in Mice. Biomaterials, 33, 2892-2901. https://doi.org/10.1016/j.biomaterials.2011.12.048

[34] Ren, H., et al. (2014) Repair of Spinal Cord Injury by Inhibition of Astrocyte Growth and Inflammatory Factor Synthesis through Local Delivery of Flavopiridol in PLGA Nanoparticles. Biomaterials, 35, 6585-6594. https://doi.org/10.1016/j.biomaterials.2014.04.042

[35] Tukmachev, D., et al. (2015) An Effective Strategy of Magnetic Stem Cell Delivery for Spinal Cord Injury Therapy. Nanoscale, 7, 3954-3958. https://doi.org/10.1039/C4NR05791K

[36] Pangestuti, R. and Kim, S.K. (2010) Neuroprotective Properties of Chitosan and Its Derivatives. Marine Drugs, 8, 2117-2128. https://doi.org/10.3390/md8072117

[37] Wu, W., et al. (2014) Neuroprotective Ferulic Acid (FA)-Glycol Chitosan (GC) Nanoparticles for Functional Restoration of Traumatically Injured Spinal Cord. Biomaterials, 35, 2355-2364. https://doi.org/10.1016/j.biomaterials.2013.11.074

[38] Dasari, V.R., Veeravalli, K.K. and Dinh, D.H. (2014) Mesenchymal Stem Cells in the Treatment of Spinal Cord Injuries: A Review. World Journal of Stem Cells, 6, 120-133. https://doi.org/10.4252/wjsc.v6.i2.120

[39] Qiu, J., Cai, D. and Filbin, M.T. (2000) Glial Inhibition of Nerve Regeneration in the Mature Mammalian CNS. Glia, 29, 166-174. https://doi.org/10.1002/(SICI)1098-1136(20000115)29:2<166::AID-GLIA10>3.0.CO; $\underline{2-G}$

[40] Goldberg, J.L. and Barres, B.A. (2000) Nogo in Nerve Regeneration. Nature, 403, 369-370. https://doi.org/10.1038/35000309

[41] Fawcett, J.W. (2006) Overcoming Inhibition in the Damaged Spinal Cord. Journal of Neurotrauma, 23, 371-383. https://doi.org/10.1089/neu.2006.23.371

[42] Liu, F., Zhang, Y., Schafer, J., Mao, G. and Goshgarian, H.G. (2019) Diaphragmatic Recovery in Rats with Cervical Spinal Cord Injury Induced by a Theophylline Nanoconjugate: Challenges for Clinical Use. The Journal of Spinal Cord Medicine, 42, 725-734. https://doi.org/10.1080/10790268.2019.1577058

[43] Lin, Y., et al. (2019) $\mathrm{NEP}_{1-40}$-Modified Human Serum Albumin Nanoparticles Enhance the Therapeutic Effect of Methylprednisolone against Spinal Cord Injury. Journal of Nanobiotechnology, 17, 12. https://doi.org/10.1186/s12951-019-0449-3 
[44] Cox, A., et al. (2020) Nanoparticle-Based Estrogen Delivery to Spinal Cord Injury Site Reduces Local Parenchymal Destruction and Improves Functional Recovery. Journal of Neurotrauma, 38, 342-352. https://doi.org/10.1089/neu.2020.7047

[45] Robinson, K., et al. (2020) A Pilot Study on the Safety of a Novel Antioxidant Nanoparticle Delivery System and Its Indirect Effects on Cytokine Levels in Four Dogs. Frontiers in Veterinary Science, 7, 447. https://doi.org/10.3389/fvets.2020.00447

[46] Vismara, I., et al. (2020) Selective Modulation of A1 Astrocytes by Drug-Loaded Nano-Structured Gel in Spinal Cord Injury. ACS Nano, 14, 360-371. https://doi.org/10.1021/acsnano.9b05579

[47] Van der Bruggen, M.A., Huisman, H.B., Beckerman, H., Bertelsmann, F.W., Polman, C.H. and Lankhorst, G.J. (2001) Randomized Trial of 4-Aminopyridine in Patients with Chronic Incomplete Spinal Cord Injury. Journal of Neurology, 248, 665-671. https://doi.org/10.1007/s004150170111

[48] Hamann, K., Nehrt, G., Ouyang, H., Duerstock, B. and Shi, R. (2007) Hydralazine Inhibits Compression and Acrolein-Mediated Injuries in ex Vivo Spinal Cord. Journal of Neurochemistry, 104, 708-718.

[49] Amani, H., Kazerooni, H., Hassanpoor, H., Akbarzadeh, A. and Pazoki-Toroudi, H. (2019) Tailoring Synthetic Polymeric Biomaterials towards Nerve Tissue Engineering: A Review. Artificial Cells, Nanomedicine, and Biotechnology, 47, 3524-3539. https://doi.org/10.1080/21691401.2019.1639723

[50] Fang, C., Ma, Z., Chen, L., Li, H., Jiang, C. and Zhang, W. (2019) Biosynthesis of Gold Nanoparticles, Characterization and Their Loading with Zonisamide as a Novel Drug Delivery System for the Treatment of Acute Spinal Cord Injury. Journal of Photochemistry and Photobiology B, Biology, 190, 72-75.

https://doi.org/10.1016/j.jphotobiol.2018.11.011 